\title{
Effects of rutin on osteoblast MC3T3-E1 differentiation, ALP activity and Runx2 protein expression
}

\author{
Xin-Wei Liu, ${ }^{1 *}$ Bin Ma, ${ }^{2 *}$ Ying Zi, ${ }^{3}$ Liang-Bi Xiang, ${ }^{1}$ Tian-Yu Han ${ }^{1}$ \\ ${ }^{1}$ Department of Orthopaedics, General Hospital of Northern Theater Command, Shenyang, Liaoning \\ ${ }^{2}$ Department of Orthopaedics, Tongji Hospital, Tongji University School of Medicine, Shanghai \\ ${ }^{3}$ Department of Emergency Medicine, Air Force Hospital of the Northern Theater of Chinese People's Liberation Army \\ (PLA), Shenyang, Liaoning, China
}

*These authors contributed equally

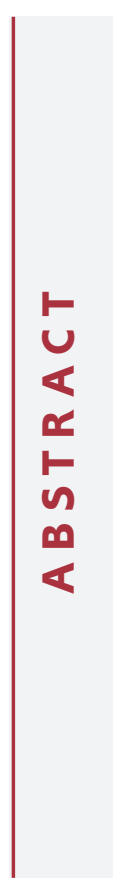

As a flavonoid, rutin has been found to have a wide range of biological functions, such as resisting inflammation and oxidation, and preventing cerebral hemorrhage and hypertension. It has been found to play an important role in osteoporosis and other orthopedic diseases in recent years. MC3T3-E1 cells were randomly divided into a control group, a rutin- 1 group $(0.01 \mathrm{mmol} / \mathrm{L})$, a rutin- 2 group $(0.05 \mathrm{mmol} / \mathrm{L})$ and a rutin-3 group $(0.1$ $\mathrm{mmol} / \mathrm{L}$ ). Osteogenic differentiation of cells was induced by osteogenic induction fluid. The control group was treated with the maximum dose of drug solvent. 2 3 days later, the solvent was replaced with fresh osteogenic induction fluid containing rutin. After a certain period of routine culture, the cells were collected for subsequent experiments. The expression of Runx2 gene in cells in all groups was detected by Real-time PCR; the expression of Runx 2 protein was detected by Western blot and immunocytochemistry (IHC); the activity of ALP was detected by reagent kit method; osteogenic differentiation was analyzed by alizarin red staining. The results of Real-time PCR showed that, compared with the control group, the treatment of cells with rutin can significantly increase the expression of Runx 2 gene $(p<0.05)$; the higher the concentration, the higher the expression of Runx 2 gene, and significant differences were found among groups in which different concentrations were used $(p<0.05)$; the results of Western blot and IHC showed that the expression trend of Runx 2 protein in each group was consistent with PCR results. In drug treatment groups, the activity of ALP was significantly higher than that in the control group $(\mathrm{p}<0.05)$; there were significant differences among groups in which different concentrations were used $(\mathrm{p}<0.05)$. The results of alizarin red staining showed that calcified nodules were formed in all groups and that the area of calcified nodules formed in groups treated with rutin was greater than that in the control group; the greater the concentration, the larger the area. Rutin can promote osteoblastic differentiation; and the greater the concentration, the more effective it is.

Key words: xRutin; Runx2; ALP; osteogenic differentiation.

Correspondence: Tian-Yu Han, Department of Orthopaedics, General Hospital of Northern Theater Command, Shenyang, Liaoning110016, China. E-mail: jwrpco@163.com

Contributions: XWL, BM, experiments concept and design; YZ, LBX, experiments performing; TYH, analysis and interpretation of experiments results. All the authors have read and approved the final version of the manuscript and agreed to be accountable for all aspects of the work.

Conflict of interest: The authors declare that they have no competing interests, and all authors confirm accuracy.

Funding: This research received no specific grant from any funding agency in the public, commercial, or not-forprofit sectors.

Availability of data and materials: All data generated or analyzed during this study are included in this published article.

Ethical Approval: The study was approved by the Institutional Ethics Committee of General Hospital of Northern Theater Command, Shenyang, Liaoning, China.

Informed consent: Not applicable. 


\section{Introduction}

Rutin is a kind of flavonoid mostly from rutin leaves, tobacco leaves and Sophora japonica buds, etc. It is widely used and has such functions as resisting inflammation and oxidation and maintaining vascular resistance. ${ }^{1,2}$ It is clinically often used to treat hypertension, prevent vascular sclerosis, cerebral hemorrhage and other diseases. ${ }^{3}$ In this study, mouse osteoblast precursor cells, MC3T3-E1 cells, were used as study subjects to explore the effects of different concentrations of rutin on the expression of Runx 2 protein and the activity of ALP during MC3T3-E1 differentiation. MC3T3-E1 cells biological functions are close to those of primary cultured osteoblasts. Therefore, they are extensively used to study the differentiation, proliferation and molecular mechanism of osteoblasts. ${ }^{4}$

During bone development, the expression of Runt family associated transcription factor 2 (Runx2) is the beginning of osteoblast differentiation. Runx 2 can initiate and activate the differentiation of bone marrow stromal stem cells into osteoblasts. Meanwhile, it can upregulate the expression of many osteogenic genes. It is a key marker in bone formation..$^{5-7}$ In addition to playing a biological role in osteoblasts, Runx2 is highly expressed in hypertrophic chondrocytes and promotes chondrocyte hypertrophy and endochondral ossification. Runx 2 also promotes osteoclast differentiation by inducing the nuclear factor $\mathrm{kB}$ receptor activator ligand (RANKL) and inhibiting the osteopontin (OPN) to play corresponding roles ${ }^{8}$. Besides, Runx 2 can also promote the synthesis of related extracellular matrix proteins, including type 1 collagen, osteocalcin (OCN) and ostesialin (BSP). ${ }^{9}$

Alkaline phosphatase (ALP) is an isoenzyme widely existing in liver, bone, placenta and other tissues. It is clinically used for the diagnosis and differentiation of rickets, malignant tumor bone metastasis, hepatobiliary diseases and other diseases..$^{10,11}$ During bone formation, ALP can hydrolyze phosphate and pyrophosphate to eliminate their inhibitory effect on bone formation, which is beneficial to further bone formation. The activity of ALP can indicate the degree of differentiation of osteoblasts and is used as one of the indicators to evaluate the mineralization ability of osteoblasts.

\section{Materials and Methods}

\section{Cell culture}

Mouse embryonic osteoblast precursor cells, MC3T3-E1 cells, were purchased from Shanghai Cell Bank, Chinese Academy of Sciences. Routine culture was performed with the $\alpha$-MEM medium containing 10\% FBS and 1\% P/S (PM150421, Procell, Wuhan, China) at $37^{\circ} \mathrm{C}$ and $5 \% \mathrm{CO}_{2}$. The state of MC3T3-E1 cells was adjusted. MC3T3-E1 cells in logarithmic phase were randomly divided into a control group, a rutin-1 group $(0.01 \mathrm{mmol} / \mathrm{L})$, a rutin-2 group $(0.05 \mathrm{mmol} / \mathrm{L})$ and a rutin-3 group $(0.1 \mathrm{mmol} / \mathrm{L})$. Osteogenic differentiation of cells was induced by osteogenic induction fluid. The control group was treated with the maximum dose of drug solvent; $2 \sim 3$ days later, the solvent was replaced with fresh osteogenic induction fluid containing rutin. After a certain period of routine culture, the cells were collected for subsequent experiments. Rutin was purchased from Solarbio Technology Co., Ltd. (SR8250; Beijing, China).

\section{Real-time PCR}

Cells were collected from all groups after 3 days of treatment with rutin. The total RNA in cell samples from each group was extracted according to the instructions for TRIpure extraction kit (RP1001; BioTek, Beijing, China). The ultraviolet spectrophotometer NanoDrop-2000 (ThermoFisher Scientific, Waltham, MA, USA) was used to determine the RNA concentration in each sample. Real-time PCR (Exicycler 96, BIONEER, Republic of Korea) was used; and super M-MLV reverse transcriptase (PR6502; BioTeke, Beijing, China) was used for reverse transcription. Realtime fluorescence quantitative PCR reaction was carried out by adding $2 \times$ Power Taq PCR MasterMix (PR1702; BioTeke), SYBR Green I (SY1020; Solarbio), cDNA template and upstream and downstream primers. Upon the completion of the reaction, B-actin was used as a reference. The formula $2^{-\Delta \Delta \mathrm{Ct}}$ was used to calculate the relative expression level of mRNA of Runx 2 gene. See Table 1 for primer sequence. It is synthesized by Sangon Biotech (Shanghai) Co., Ltd. (Shanghai, China).

\section{Western blot}

The cells in all groups were treated with rutin for 3 days. The treated cells were split with the RIPA lysis buffer (P0100; Solarbio) on the icebox for $5 \mathrm{~min}$; then the cells were centrifuged at $12,000 \mathrm{rpm}$ and $4^{\circ} \mathrm{C}$ for $10 \mathrm{~min}$. The supernatant was separated to obtain protein extract. BCA protein assay kit (WLA004, Wanleibio, Shenyang, China) was used for quantitative analysis of the total protein (TP) extracted to get protein concentration. The electrophoresis apparatus was assembled and polyacrylamide gel prepared. 8\% SDS-PAGE was used for electrophoresis. After electrophoresis, the protein bands separated were transferred to the PVDF membrane (IPVH00010; Millipore, Burlington, MA, USA). The PVDF membrane was sealed with 5\% skim milk; then Runx2 primary antibody (diluted by 1:500; WL03358; Wanleibio) was added. The PVDF membrane was incubated at $4{ }^{\circ} \mathrm{C}$ overnight. Upon the completion of incubation, the PVDF membrane was washed with TBST. The horseradish peroxidase (HRP)-labeled sheep anti-rabbit IgG (diluted by 1:5000; SE134; Solarbio) was added for incubation. The reference $\beta$-actin was incubated by the same experimental procedures. Finally, ECL luminous fluid (PE0010; Solarbio) was added for exposure in a darkroom. The film was scanned. The gel image processing system (the software Gel-Pro-Analyzer) was used to analyze the optical density of the target band.

\section{Immunocytochemistry (IHC)}

After 3 days of treatment with rutin, the cells treated in each group were made into cell slides. Four percent paraformaldehyde solution was used for fixation for $15 \mathrm{~min}$. Next, $0.1 \%$ TritonX-100 was added for incubation at ambient temperature for $20 \mathrm{~min}$ and then discarded. $3 \% \mathrm{H}_{2} \mathrm{O}_{2}$ was added for incubation. The serum was added for sealing for $15 \mathrm{~min}$. The serum was discarded, the Runx2 primary antibody diluted with PBS (diluted by 1:500; WL03358;

Table 1. Primer sequence.

\begin{tabular}{lrr} 
Name of primer & Former primer $(5-3)$ & Back primer $(5-3)$ \\
Runx2 & GCAGCACTCCATATCTCTACT & TTCCGTCAGCGTCAACAC \\
$\beta$-actin & CTGTGCCCATCTACGAGGGCTAT & TTTGATGTCACGCACGATTTCC \\
\hline
\end{tabular}


Wanleibio) was added. The mixture was placed at $4^{\circ} \mathrm{C}$ overnight. The primary antibody was discarded. PBS was used to dilute the HRP-labeled secondary antibody by 1000 times (diluted by 1:1000; \#31460, ThermoFisher). The mixture was added to completely cover the cells. The cells were incubated at $37^{\circ} \mathrm{C}$ for $1 \mathrm{~h}$. The secondary antibody was removed. Hematoxylin was used for redyeing (G1004-100; Servicebio, Wuhan, China). Sealing was performed. The immunopositivity was observed and photographed under the microscope at 400x magnification. The software ImagePro Plus was used for optical density analysis.

\section{Determination of ALP activity}

After the treatment of cells with rutin, the activity of ALP in cells in each group was detected according to the instructions for ALP activity detection kit (WLA064; Wanleibio).

\section{Alizarin red staining}

After 14 days of rutin intervention, alizarin red staining was performed to analyze the osteogenic differentiation of cells in different treatment groups. The medium in each group was removed. $4 \%$ paraformaldehyde was used for fixation at ambient temperature for $15 \mathrm{~min}$. The fixative was discarded. Cells were washed with distilled water for 3 times. Alizarin red S (G1450; Solarbio) was added for staining for $30 \mathrm{~min}$. The staining solution was removed. Cells were washed with distilled water for 3 times again. The magnification of the microscope was adjusted to 100 times for photographing and analyzing.

\section{Statistical analysis}

The data were analyzed with SPSS 20.0. All results were expressed as means $\pm \mathrm{SD}$. The counting data were tested by chisquare test; the difference was deemed statistically significant if $\mathrm{p}<0.05\left({ }^{*} \mathrm{p}<0.05,{ }^{* *} \mathrm{p}<0.01, * * * \mathrm{p}<0.001\right)$. The software GraphPad Prism 8.0 was used to draw charts.

\section{Results}

\section{Effects of rutin on expression of Runx2 gene in cells}

After 3 days of treatment of MC3T3-E1 cells with different concentrations of rutin, the expression of Runx2 gene was detected by PCR. The results showed that, compared with the control group, 3 concentrations of rutin could significantly increase the expression quantity of Runx2 gene, and there were significant differences $(p<0.05)$. The relation between different concentrations of rutin was compared; and it was found that the greater the concentration, the higher the expression quantity of Runx 2 gene. There were highly significant differences among the 3 concentrations $(\mathrm{p}<0.05)$ (Figure 1)

\section{Effects of rutin on expression of Runx2 protein in cells}

After the treatment of cells in all groups with rutin for 3 days, the expression of Runx 2 protein was detected by Western blot (WB) and IHC, respectively. WB results showed that rutin can significantly increase the expression level of Runx 2 protein, and the difference was statistically significant; with the increase of rutin concentration. The expression level of Runx 2 protein also got higher and higher, and there were significant differences $(p<0.05)$. The IHC results showed that, there was no significant difference between the low concentration group and the control group. The comparative results among other groups were consistent with those of WB (Figure $2 \mathrm{~A}, \mathrm{~B})$.

\section{Effects of rutin on ALP activity in cells}

The ALP activity was detected with ALP activity detection kit after the cells in all groups were treated with rutin for 5 days. It was found that, compared with the control group, the ALP activity of all treatment groups was significantly improved, and the greater the concentration, the higher the ALP activity $(\mathrm{p}<0.05)$; and there were significant differences in the ALP activity among different concentration groups $(\mathrm{p}<0.05)$ (Figure 3 ).

\section{Detection of osteogenic differentiation of cells by alizarin red staining}

The differentiation in cells was analyzed by alizarin red staining after 14 days of treatment with rutin. The results showed that calcified nodules were formed in all groups and that the area of calcified nodules formed in groups treated with rutin was greater than that in the control group; the greater the concentration of rutin, the larger the more obvious the nodules (Figure 4).

\section{Discussion}

Rutin is a flavonoid found in many plants, has a wide range of biological activities, and can be used to resist oxidation and inflammation, protect the nerve and liver, and prevent cerebral hemorrhage. ${ }^{12}$ In addition to some diseases that have been known and clinically treated with rutin, the role of rutin in other diseases has also been gradually taken seriously by people. Previous studies showed that rutin has important potential in the prevention and treatment of diabetes. ${ }^{13,14}$ The effects of rutin in orthopedic diseases have also been gradually known by people. Osteoporosis is a chronic disease; the main cause is that the bone has lost its mineralization quality and mechanical flexibility. Since the currently used anti-osteoporosis agents have some efficacy but also some side effects, including an increased risk of blood clots and cancer, phytochemicals may be a safer and more effective option. The

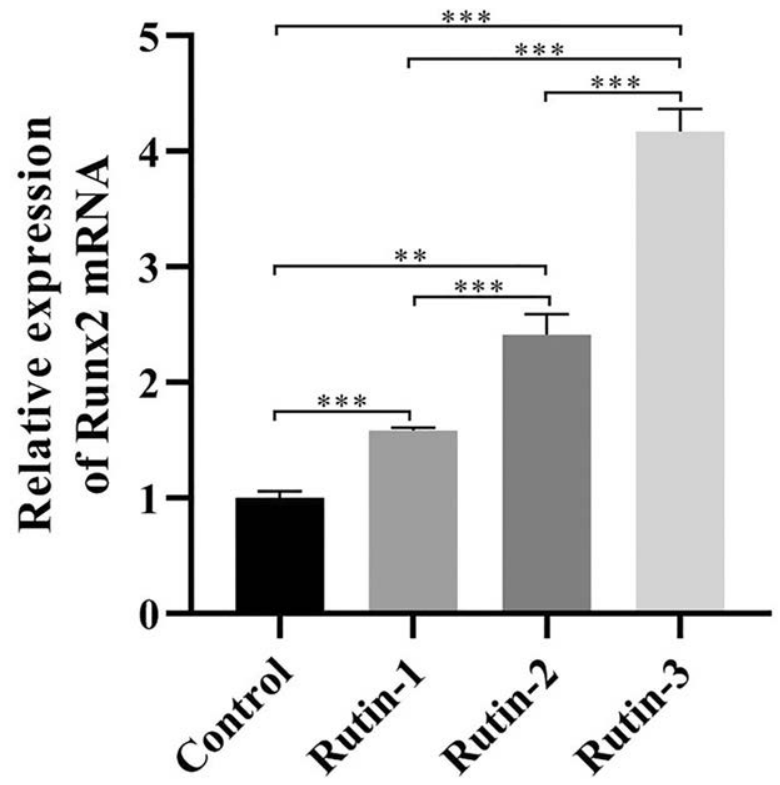

Figure 1. Expression of Runx2 gene after treatment of MC3T3-E1 cells with different concentrations of rutin. 
study on human osteosarcoma cells, SAOS-2 cells, showed that rutin can increase the expression of osteocyte and osteoblast-related genes and decrease the expression of Runx inhibitor and osteoclast gene. The study on the effects of rutin on ALP activity demonstrated that rutin can improve ALP activity and decrease the activity of acid phosphatase, a marker for osteoporosis. Therefore, rutin can promote the proliferation of osteocyte and act as an ossification marker. ${ }^{15}$ The results of this study are consistent with those of our study, proving that rutin can be used as an effective drug for future treatment of orthopedic diseases. Xiao et al. explored the mechanism of action of rutin in osteoporosis and found by analyzing by multiple experimental methods, including Micro-CT, Western-Blotting, Real-time PCR, transmission electron microscope and alizarin red staining, that rutin can regulate FNCD1 level and autophagy through the Akt/mTOR signaling pathway, which provides a new strategy for the treatment of osteoporosis. ${ }^{16}$ Rutin can also protect human periodontal ligament stem cells (HPDLSCs) from the TNF- $\alpha$-induced osteogenic differentiation damage in an inflammatory environment, and is expected to be a new candidate drug for the treatment of periodontitis bone defects. ${ }^{17}$

The formation and reconstruction of bone is a complicated process, including osteoblasts forming new bone and osteoclasts absorbing old bone to maintain a dynamic balance. Runx2, also known as core-binding factor al, is one of the important members of the Runt family. The other two members are Runt1 and Runx3. Some studies have shown that, in the early stage of osteoblast differentiation, Runx2 gene can trigger the synthesis of bone matrix protein and provide a great number of immature osteoblasts. ${ }^{18}$ It can be used as a specific marker gene to indicate osteoblast differentiation and bone formation. Moreover, Runx2 gene activates the transcription and expression of bone sialoprotein, osteopontin and type I collagen gene by binding to cis-acting elements of osteoblasts. ${ }^{19}$ The molecular osteoprotegerin (OPG) produced by osteoblasts plays an important role in the formation of osteoclasts; OPG can not only inhibit the formation of osteoclast-like cells in

A
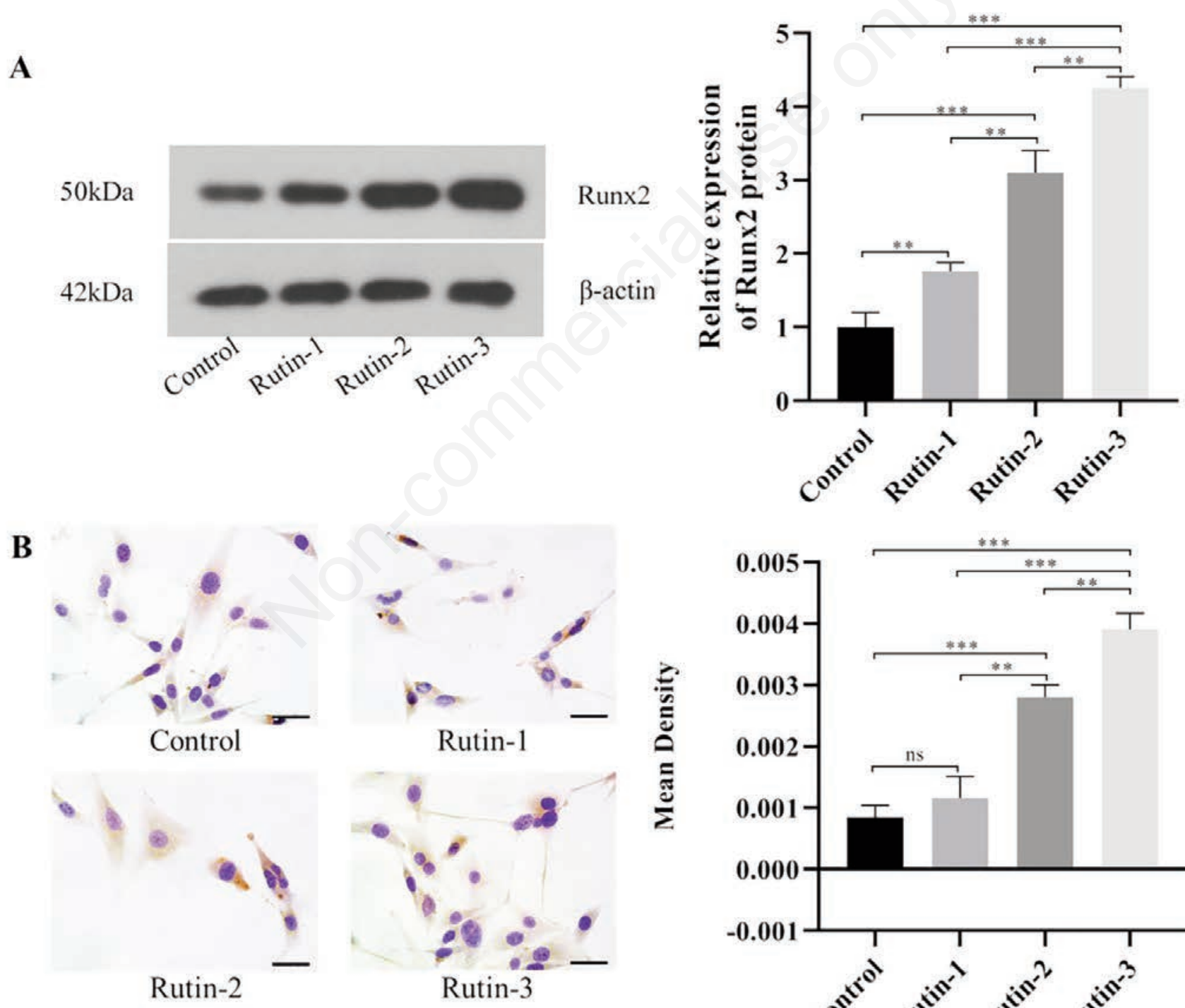

Rutin-2

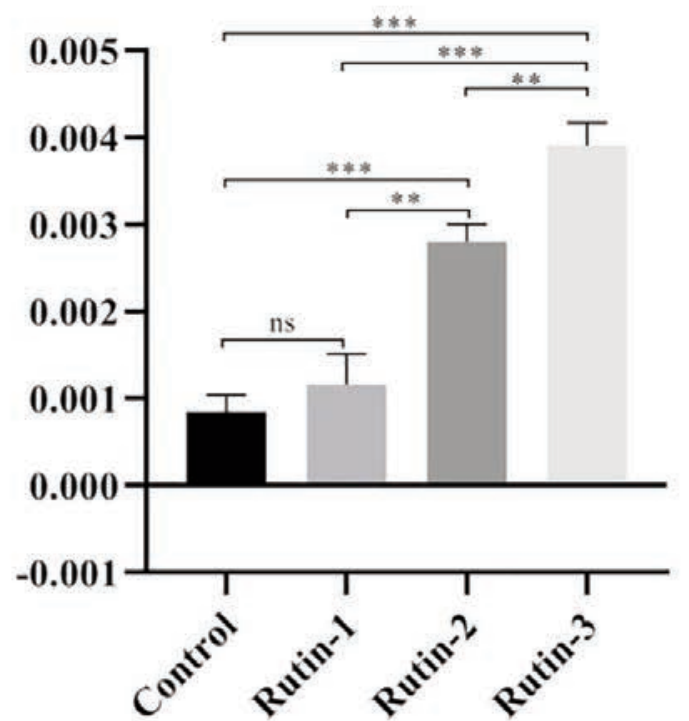

Figure 2. Expression of Runx2 protein in cells after treatment with rutin. A) Western blot; B) Immunohistochemistry. 


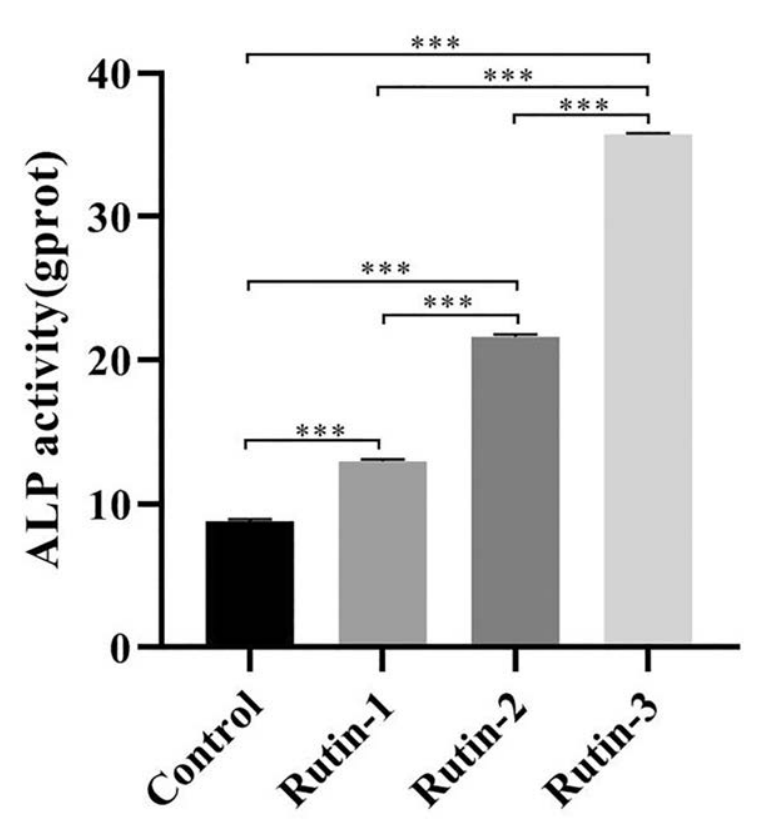

Figure 3. ALP activity after treatment of cells with rutin. vivo and in vitro but also absorb bone. The sequence cloning of human OPG showed that there is a binding site for OPG in Runx2 gene sequence, indicating that Runx2 can regulate OPG expression. ${ }^{9,20}$ Runx 2 gene is highly expressed in multiple myeloma cells and is one of the major drivers of myeloma progression. Lowering the abundance of Runx2 by regulating the upstream genes of Runx 2 can slow down the progression multiple myeloma. ${ }^{21}$ The results are consistent with ours. With the deepening of study and the understanding of the richer functions and mechanism of action of Runx 2 gene, it will certainly provide a detailed theoretical basis and practical significance for the treatment of bone-related diseases.

ALP is a specific enzyme secreted by osteoblasts. Its activity directly reflects the degree of the differentiation of osteoblasts. Zhang et al. confirmed that ALP was highly expressed in both in vitro osteogenic differentiation and in vivo bone formation. ${ }^{22}$ Similar to rutin, astragalin is also a bioactive flavonoid mostly from medicinal plant. After the treatment of MC3T3-E1 cells with astragalin, high expression of ALP was also detected and significantly increased; besides, the expression level of Runx 2 was also higher than that in the control group. The results are consistent with those of this study. ${ }^{23}$

It is believed that, with the deepening of study, rutin will play a better role in the treatment of orthopedic diseases, with great significance for their cure.

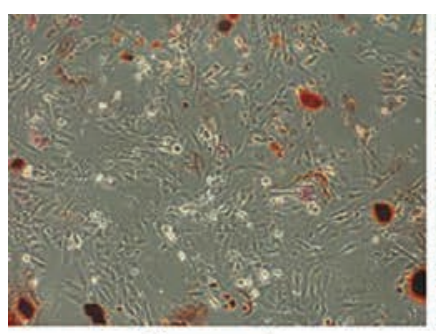

Control

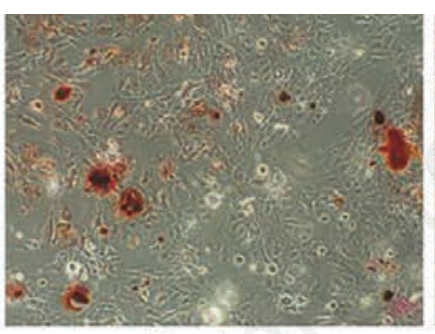

Rutin-1

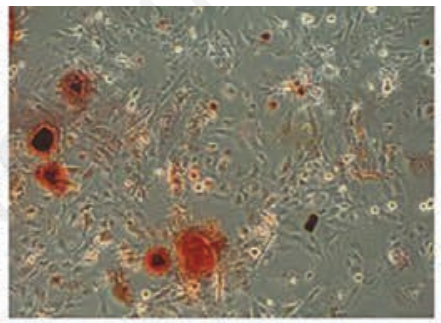

Rutin-2

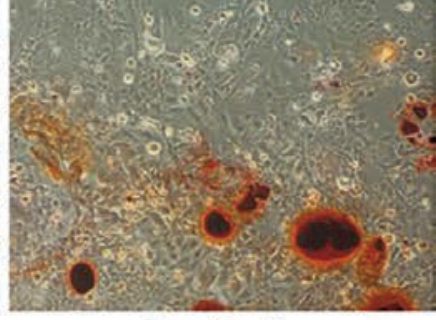

Rutin-3

Figure 4. Analysis of osteogenic differentiation of cells by alizarin red staining.

\section{References}

1. Solomon H. Rutin as a natural therapy for Alzheimer's disease: Insights into its mechanisms of action. Curr Med Chem 2016;23:860-73

2. Solomon H, Abebech B. Natural therapies of the inflammatory bowel disease: The case of rutin and its aglycone, quercetin. Mini Rev Med Chem 2018;18:234-43.

3. Lee S C.A review on plant-based rutin extraction methods and its pharmacological activities. J Ethnopharmacol 2013;150: 805-17.

4. Fushimi S, Nohno T, Nagatsuka H, Katsuyama H. Involvement of miR-140-3p in Wnt3a and TGF 33 signaling pathways during osteoblast differentiation in MC3T3-E1 cells. Genes Cells 2018;23:517-27.

5. Buo AM, Tomlinson RE, Eidelman ER, Chason M, Stains JP. Connexin43 and Runx2 interact to affect cortical bone geometry, skeletal development, and osteoblast and osteoclast function. J Bone Miner Res 2017;32:1727-38.

6. Toshihisa K. Regulation of bone development and mainte- nance by Runx2. Front Biosci 2008;13:898-903.

7. Luca DC, Giulio I, Maria TV, Dalle Carbonare L, Innamorati G, Valenti MT. Transcription factor Runx2 and its application to bone tissue engineering. Stem Cell Rev Rep 2012;8:891-7.

8. Kyung TW, Lee JE, Shin HH, Choi HS. Rutin inhibits osteoclast formation by decreasing reactive oxygen species and TNF-alpha by inhibiting activation of NF-kappaB. Exp Mol Med 2008;40:52-8.

9. Toshihisa K. Roles of Runx2 in skeletal development. Adv Exp Med Biol 2017;962:83-93.

10. Birmingham E, Niebur GL, McHugh PE, Shaw G, Barry FP, McNamara LM. Osteogenic differentiation of mesenchymal stem cells is regulated by osteocyte and osteoblast cells in a simplified bone niche. Eur Cell Mater 2012;23:13-27.

11. Zhang L, Gong Z X. Clinical characteristics and prognostic factors in bone metastases from lung cancer. Med Sci Monit 2017;23:4087-94.

12. Ravi GS, Charyulu RN, Dubey A, Prabhu P, Hebbar S, Mathias AC. Nano-lipid complex of rutin: Development, characterisation and in vivo investigation of hepatoprotective, antioxidant 
activity and bioavailability study in rats. AAPS PharmSciTech 2018;19:3631-49.

13. Ghorbani A. Mechanisms of antidiabetic effects of flavonoid rutin. Biomed Pharmacother 2017;96:305-312.

14. Habtemariam S, Lentini G. The therapeutic potential of rutin for diabetes: an update. Mini Rev Med Chem 2015;15:524-8.

15. Abdel-Naim AB, Alghamdi AA, Algandaby MM, Al-Abbasi FA, Al-Abd AM, Eid BG, et al. Rutin isolated from chrozophora tinctoria enhances bone cell proliferation and ossification markers. Oxid Med Cell Longev 2018;2018:5106469.

16. Xiao Y, Wei R, Yuan Z, Lan X, Kuang J, Hu D, et al. Rutin suppresses FNDC1 expression in bone marrow mesenchymal stem cells to inhibit postmenopausal osteoporosis. Am J Transl Res 2019;11:6680-90.

17. Zhao B, Zhang W, Xiong Y, Zhang Y, Jia L, Xu X. Rutin protects human periodontal ligament stem cells from TNF- $\alpha$ induced damage to osteogenic differentiation through suppressing mTOR signaling pathway in inflammatory environment. Arch Oral Biol 2020;109:104584.

18. Toshihisa K. Regulation of osteoblast differentiation by tran- scription factors. J Cell Biochem 2006;99:1233-9.

19. Ducy P, Starbuck M, Priemel M, Shen J, Pinero G, Geoffroy V, et al. A Cbfa1-dependent genetic pathway controls bone formation beyond embryonic development. Genes Dev 1999;13:1025-36.

21. Toshihisa K. Regulation of proliferation, differentiation and functions of osteoblasts by Runx2. Int J Mol Sci 2019;20:1694.

22. Gowda PS, Wildman BJ, Trotter TN, Xu X, Hao X, Hassan $\mathrm{MQ}$, et al. Runx2 suppression by miR-342 and miR-363 inhibits multiple myeloma progression. Mol Cancer Res 2018;16:1138-48.

23. Zhang J, Zhang W, Dai J, Wang X, Shen SG. Overexpression of Dlx2 enhances osteogenic differentiation of BMSCs and MC3T3-E1 cells via direct upregulation of osteocalcin and ALP. Int J Oral Sci 2019;11:12.

24. Liu L, Wang D, Qin Y, Xu M, Zhou L, Xu W, et al. Astragalin promotes osteoblastic differentiation in MC3T3-E1 cells and bone formation in vivo. Front Endocrinol (Lausanne) 2019;10:228. 